\title{
GovML: A Markup Language for Describing Public Services and Life Events
}

\author{
Gregory Kavadias and Efthimios Tambouris \\ Archetypon S.A., 236 Sygrou Av., Athens, 176-72, Greece \\ \{gkavadias, tambouris\} @archetypon.gr \\ http: //www.archetypon.gr/egov/
}

\begin{abstract}
The Governmental Markup Language (GovML) has been proposed by the IST eGOV project to define structures (or vocabularies) for governmental data and metadata. In this paper, the GovML data vocabularies for describing public services and life events are presented. Technically, GovML is a proposed format for XML documents. Its adoption by public authorities will allow users (citizens, businesses) of public services to exploit the potential of XML technology. For example, users will be able to access governmental information in a unified presentation style through multiple communication channels (web browsers, mobile phones, handheld devices etc).
\end{abstract}

\section{Introduction}

E-government is becoming increasingly important for all European Union Member States. A large number of e-government initiatives and projects are currently under way, aiming to enhance access and delivery of government services to benefit citizens, business partners and employees [3]. Public authorities are providing a number of public services online. At the presentation layer, public services are grouped based on users needs and intentions e.g. in life events. Consequently, there is a substantial amount of information describing public services and life events.

The main idea behind this paper is that the information describing public services and life events could be standardised. We believe that public organisations and consumers of public services could both benefit from a standard electronic format for the description of public services. Public authorities would have a common structure for describing all their services, thus production and management of governmental information would be eased, while interoperability with other agencies would be fostered. On the other hand, users would more easily navigate through different public services. The more structured the information is, the more easily a human can understand it and use its information to make knowledgeable decisions

In this paper the Governmental Markup Language (GovML) data vocabulary is presented as a proposed document structure for the public sector. GovML is the result of research work conducted within the IST eGOV project [9]. GovML aims to overcome the arbitrary principles applied to the structure and presentation of governmental content at an international level.

From a technical point of view, GovML data vocabularies are a tailored XML implementation, suggesting a common XML structure for public services descriptions

The original version of this chapter was revised: The copyright line was incorrect. This has been corrected. The Erratum to this chapter is available at DOI: 10.1007/978-3-540-44836-5_33

M.A. Wimmer (Ed.): KMGov 2003, LNAI 2645, pp. 106-115, 2003.

(C) Springer-Verlag Berlin Heidelberg 2003 
and life events. By adopting GovML, users (citizens and businesses) are given the opportunity to access governmental information through multiple access channels. Moreover, GovML is characterised by flexibility and extensibility, thus facilitating its maintenance and adaptation to potential new requirements.

The rest of this paper is organised into 6 sections. In section 2 some background information is given, while in section 3 the motivation for the invention of GovML is explained. In section 4 the methodology and its outcome (i.e. the specified vocabularies for public services descriptions and life events) are presented. In section 5 an overview of the technical implementation and the utilised XML schema mechanisms are analysed, along with an example and a usage scenario. Finally in section 6 the main conclusions are presented.

\section{Background}

\subsection{The IST eGOV Project}

The work reported in this paper was carried out within the eGOV project. eGOV is a two-years EC-funded RTD project within the 5th Framework Program of the European Commission. The main objective of the project is to specify, develop, deploy and evaluate an integrated platform for realising online one-stop government. The eGOV platform will allow the public sector to provide citizens with information and services, based on 'life-events'. This platform will be deployed and evaluated in Austria and Greece.

The main technical objectives of the eGOV project include the specification and development of:

- The next generation of online one-stop governmental portals and the supporting network architecture. This sort of portals will feature a number of advanced characteristics: access from different devices including WAP-enabled devices, personalisation, customisation, multilinguality, support of push services etc.

- The Governmental Markup Language (GovML), an open XML document structure aiming to support data exchange among public agencies and their consumers.

Qualitative, effective and efficient access to governmental information is a critical success factor of e-government. The eGOV project aims to address this requirement by providing an integrated platform for one-stop government, along with the supporting process models.

\subsection{Life Event Approach to Access of Governmental Information}

The concept of "life events" is an increasingly used guiding metaphor for accessing public services and information online. Within the eGOV project, life events are defined as follows:

"Life events describe situations of human beings that trigger public services." Examples of life events are: getting married, traveling abroad etc.

In a similar way, "business situations describe topics of companies and selfemployed citizens that trigger public services or interactions with public authorities." 
Examples of business situations are: founding a company, (re-) constructing factory premises etc.

Life events and business situations can be used as meaningful metaphors at the presentation layer, aiming to facilitate and enhance users' access to governmental information and services. This approach gives users the opportunity to disregard the complexity and functional fragmentation of the public sector [16]. For example, the "getting married" life-event, ideally should enable citizens to electronically handle all possible interactions with the public agencies.

\section{Motivation for Work}

Knowledge management can be perceived as "how an organisation can get its arms around all of the knowledge and information held in the hundreds of gigabytes of information in the organisation's computer systems, e-mail systems, word documents, spreadsheets and so on, in order to meet its objectives" [13].

Unless organized, information provided by public authorities cannot be retrieved and disseminated to the consumers of public services

Thus, the demand for knowledge management within public authorities necessitates the creation of standard templates for describing information provided by public authorities.

Most of public agencies at an international level, make an effort to offer a significant portion of their services online. In this respect, citizens and businesses can benefit from Internet technology, as they can be electronically informed and interact with the public sector. Provided with remote access to public information and services, consumers are given the opportunity to avoid queuing, losing time for moving to several public authorities, finding contact persons, etc.

Online information however, does not comply with a specific structure. Furthermore, it is not characterized by unified presentation style and principles. On the contrary, it is very common that each public authority applies its own format to the presentation of information, hence preventing users from a unique experience, whenever they interact with the public sector.

Apart from presentation of pubic information, another important issue in online one-stop government is interoperability. It is common in e-government projects that several governmental data need to be exchanged among public authorities. Each authority however, usually stores governmental information in a proprietary data format and storing system e.g. HTML, ASCII, RDBMS etc. Therefore data conversion must take place from the format adopted by one public authority to the format adopted by others, whenever exchange of data is needed. This data conversion can become a significant overhead to the Information Technology (IT) responsibilities of public authorities.

The evolution of mobile phones and handheld devices are another challenge when implementing online one-stop government through governmental portals. It is common that public authorities wish to provide their users with access to governmental information and services through multiple communication channels: web browsers, mobile phones, Personal Digital Assistants (PDA) etc. This allows governmental information and services to be available 24 hours a day, via multiple access devices from everywhere, even on the move. 


\section{Towards Identification of GovML Elements}

The proposed Governmental Markup Language (GovML) addresses the common structure, presentation and interoperability challenges stated in section 3 . The eGOV consortium consists of 10 partners coming from Austria, Finland, Germany, Greece and Switzerland. Partners represent a mixture of private IT companies, academic and research institutes and public administrations. All parties collaborated closely in order to determine the components of the proposed document structure, for the description of public services and life events. The steps that were followed towards the specification of GovML data elements are (Figure 1):

1. All partners performed an analysis of existing governmental portals and Web sites. Existing governmental sites at an international level [1], [2], [7], [14], [15], [17], [18] were investigated and best practices of online eGovernment were taken into account. The investigation focused on structure and presentation of the public sector content, from the users' point of view.

2. Public services provided by various public organisations were investigated. Each partner of the eGOV consortium investigated a number of public services that were provided not only online but also non electronically. Subsequently, the necessary elements needed to describe the investigated public services and life events were identified.

3. The contributions of the eGOV consortium's partners were assembled and the first version of the proposed GovML data elements was circulated among the partners.

4. All partners commented on the first version of GovML data elements and proposed refinements. All comments were gathered and evaluated. Thereafter a new version of the GovML data elements was produced and circulated to the eGOV consortium for consultation.

5. Iteration among steps 3 to 5 was realised until consensus was reached between all partners of the eGOV consortium.

6. The final GovML data elements were reported according to ISO/IEC 11179-3 standard [8].

The final GovML data structure consists of three vocabularies, two for describing public services and one for life events. Technically, each sub-vocabulary is characterized by a set of predefined XML elements. The three GovML vocabularies are analysed in the next paragraphs.

\section{Generic description data vocabulary for public services}

This vocabulary defines a common standard for the content of all public authorities at a national level. Such governmental content is created only once, at a national level. This type of content could be normally based upon a governmental law, so it can be adopted by all public agencies of a country. Examples of data elements are: title, procedure, required documents, etc.

\section{Specific description data vocabulary for public services}

This vocabulary caters for the creation of content related to a public service provided by a specific public authority. It can be considered as a specialization of the generic description vocabulary, because the values of some elements of this vocabulary 
depend on the public authority, which provides the public service. Some of its elements are: name and address of the public authority, public servant contact details, delivery channel of the service, etc.

It should be noted that the generic and specific data vocabularies for public services have many common elements.

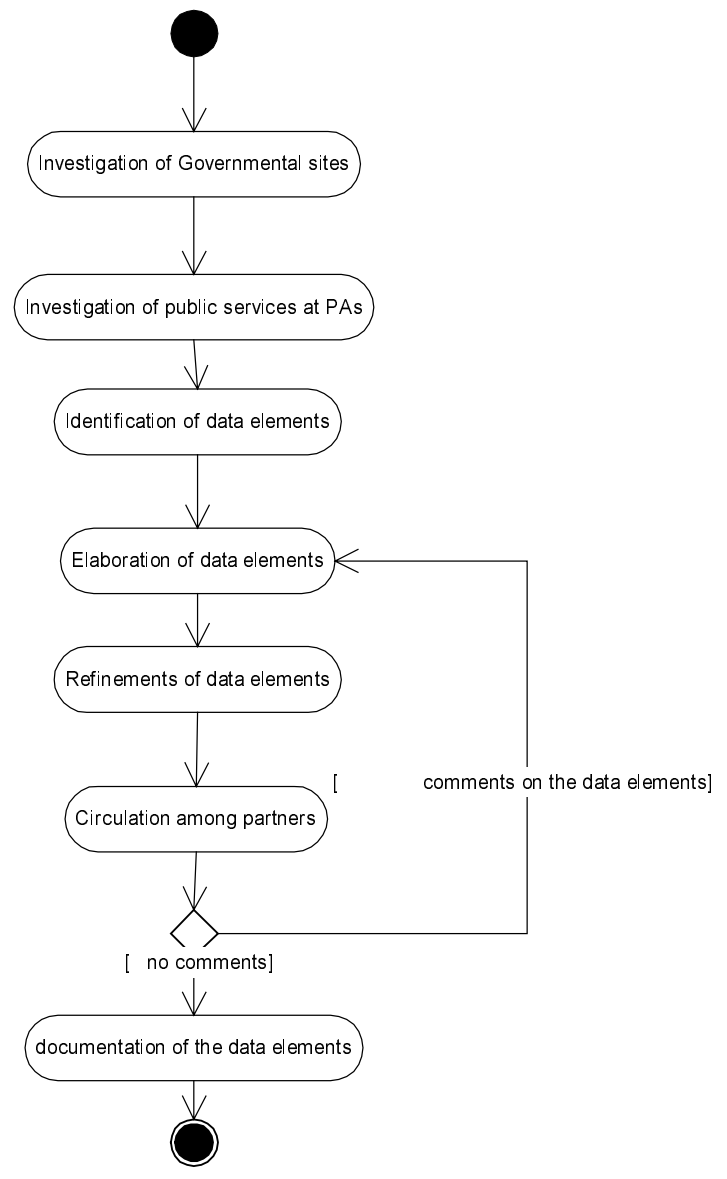

Fig. 1. Methodology towards the identification of the data elements

\section{Data vocabulary for life events and business situations}

This vocabulary defines a set of elements necessary to describe any every day life event or business situation. Elements of this vocabulary are a subset of the generic description data vocabulary for public services. The three GovML data vocabularies are listed in Table 1. 
Table 1. GovML data vocabulary

Public Services

Life Events

\begin{tabular}{cccc}
\hline & Generic description & Specific description & Description \\
\hline 1 & identifier & identifier & identifier \\
\hline 2 & language & language & language \\
\hline 3 & title & title & title \\
\hline 4 & description & description & description \\
\hline 5 & attention & attention & attention \\
\hline 6 & faq-list & faq-list & faq-list \\
\hline 7 & eligibility & eligibility & \\
\hline 8 & required-documents & required-documents & \\
\hline 9 & procedure & procedure & \\
\hline 10 & periodicity & periodicity & \\
\hline 11 & time-to deliver & time-to deliver & \\
\hline 12 & cost-Info & cost-Info & \\
\hline 13 & service-hours & service-hours & \\
\hline 14 & employee-hints & employee-hints & \\
\hline 15 & citizen-hints & citizen-hints & \\
\hline 16 & related-services & public-authority-name & related-services \\
\hline 17 & audience & Public authority department & \\
\hline 18 & public-authority-type & e-documents \\
\hline 19 & law & delivery-channel \\
\hline 20 & result & cost & \\
\hline 21 & \multicolumn{4}{c}{ delivery-channel } \\
\hline 22 & \multicolumn{4}{c}{ contact-details } \\
\hline 23 & \multicolumn{4}{c}{ service-code } \\
\hline 24 & automation-level \\
\hline 25 & public-authority-address \\
\hline 26 & state \\
\hline
\end{tabular}

\section{Implementation of GovML}

\subsection{XML Schema of GovML}

From a technical point of view, the Governmental Markup Language (GovML) vocabularies are implemented using XML technologies. More specifically, in order to serialize GovML documents in XML format, an XML schema was implemented for the validation of their structure. The XML schema validation mechanism (http://www.w3.org/XML/Schema) was preferred from Document Type Definition (DTD) because it provides a richer set of data-types (including byte, date, integer etc) and allows users to derive their own data types and take advantage of inheritance of elements, attributes and definitions of data-types [10].

New XML documents describing public services or life events can emerge from the XML schema of GovML. Consequently, the appropriate XSL Transformations (XSLT) [19] should be applied for transforming GovML documents to the required format (HTML, WML etc).

XML schema can be easily extended, modified and maintained in the future according to consumer's needs. The full XML schema of GovML can be found in [2]. 


\subsection{Example of a GovML Document}

A GovML document describing a life event is illustrated in Figure 2. This document is valid against the XML schema of GovML. Data elements described in the third column of Table 1, are serialized in this sample GovML document. Hence this document is uniquely identified by the identifier element and the language of the document is characterised by the language element. In this example language is English (EN). Title and description elements are two descriptive fields. The attention field contains what the user should pay attention at and the faq-list is a list of the most frequent asked questions of users along with their answers. Related-Services includes public services related to the described life event along with their links in form of URI. Law is an optional element describing the governmental law related to the described life event.

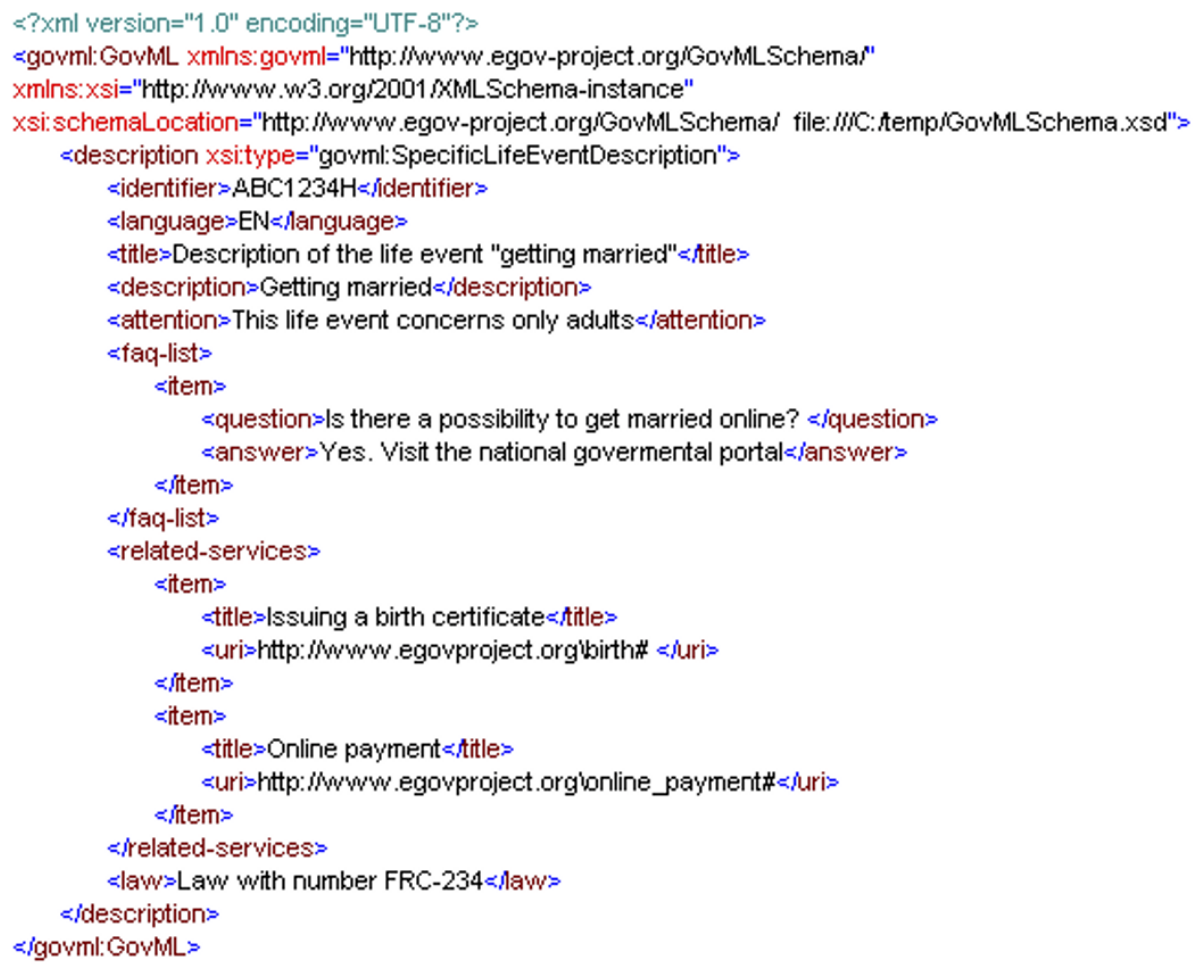

Fig. 2. Example of a GovM document describing a life event

\subsection{GovML's Document Structure and XML Schema Complex Types}

In implementation terms, the three GovML data vocabularies (generic service description, specific service description and generic life events description) are based on a number of XML schema complex types. Examples of such complex types are: 
the "address" complex type used to describe the address of a public organisation and "contact details" used to describe the details of a contact person at a public agency.

Each of the three GovML data vocabularies has been built on a hierarchy of complex types. Hence GovML vocabularies are combinations of a number of complex types in a predefined order. A complex type named "GovMLDescription" serves as a base type that all data vocabularies (Generic and Specific service description and life event description) inherit from. The GovMLDescription base type is illustrated in Figure 3.

Language, title and description are mandatory XML elements for any type of GovML document.

GovML structures take advantage of re-usability and extensibility, which characterise XML schema complex types. As a result, GovML defines data structures, which are open, flexible, extensible end easy to maintain.

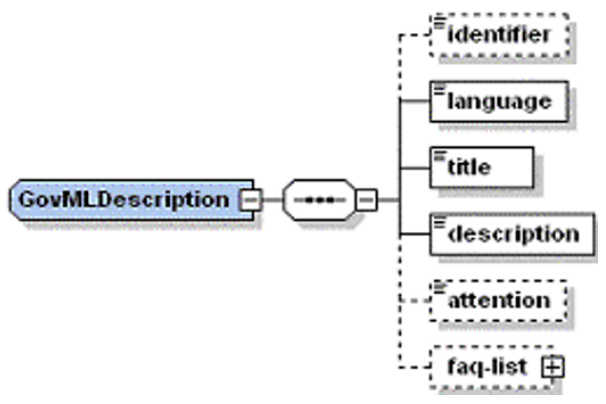

Fig. 3. GovMLDescription complex type

\subsection{Lifecycle of a GovML Document According to eGOV Project}

Certain processes at public authorities support the production and provision of GovML documents. In order to demonstrate the life cycle of a GovML document, a simplified scenario is described. It covers all stages, from document creation until its eventual depiction to consumers.

Supposing that a public authority is planning to provide citizens with the ability to obtain a birth certificate online. Firstly, a public servant is assigned with the administrator role. At a next step, the description of the public service should be generated in GovML. For this purpose, a web application named Service Description Tool (SDT) is utilised. SDT has been implemented within the eGOV project, to enable the management of GovML documents. SDT enables administrators of eGOV platform at public agencies to create, retrieve, update and delete XML documents via a user-friendly Graphical User Interface, following a sequence of predefined steps. Advanced XML techniques (i.e. indexing mechanisms based on Xpath expressions) are applied in order to enhance the efficient manipulation of GovML documents. SDT is a multilingual application, currently supporting English, German, French and Greek. 
By using SDT, the administrator fills out the elements of the new GovML document. Their content derives from the related governmental law, rules at a national level and the regulations of the public authority (in case of a specific GovML). In this example, it is assumed that the administrator produces a specific service description. The element identifier aims to uniquely identify the GovML for internal management reasons (i.e. storage, retrieval etc). It is automatically filled in by the SDT. Language is an element of type xml:lang, illustrating the language of the document. It is also filled in by the system implicitly, after the administrator's language choice from the SDT. Title and description describe the title and a short description of the provided public service respectively. Eligibility element explains which citizens have the right to consume the public service i.e. who can apply for issuing a birth certificate. Required-documents element provides the list of transcripts that are considered prerequisite for the provision of the service; in our example these could be passport or identity card. Procedure describes analytically the steps that the consumer has to follow and periodicity illustrates how often the service is available. In this scenario it could have the value "every working day".

In a similar way the administrator fills in the rest of the data elements illustrated in the second column of Table 1 and stores the new GovML document via SDT. The new document is stored in the local repository of the public organisation, which contains all GovML documents of this organization.

The final step is to transform the document to a specific display format e.g. HTML or WML. This can be achieved with the utilisation of an appropriate XSLT script on the public authority side. The transformation mechanism is transparent to the users who are given the opportunity to access the desired information in multiple formats, for example HTML through a web browser or WML through a mobile phone supporting the WAP protocol.

\section{Conclusions}

In this paper the data vocabularies of Governmental Markup Language (GovML) were presented. GovML data vocabularies propose data structures for the description of public services and life events. GovML provides a uniform data structure that enables standardisation of public information aiming at the satisfaction of users by making information more accessible.

The better structured the information in a document is, the more easily a human can understand it, which in turn means that the human can make use of that information in order to make knowledgeable decisions. Hence, the standardization of governmental documents is a significant enabling technology for Knowledge Management.

It is anticipated that the wide adoption of GovML by public authorities will facilitate the exchange of information between public authorities and will enhance the experience of users (citizens, businesses) when accessing information provided by public organisations. For example, users will be able to access governmental information in a unified presentation style through multiple communication channels (web browsers, mobile phones etc). 
Acknowledgements. The eGOV project has commenced on the 1st of June 2001 and is co-funded by the European Commission under contract IST-2000-28471. The authors would like to acknowledge the assistance of all partners of the eGOV consortium in deriving GovML. The partners of the eGOV consortium are: Siemens Austria (financial coordinator); Archetypon S. A. (administrative and scientific coordinator); TietoEnator Corporation; IKV ++, University of Linz; NCSR Demokritos; Hellenic Ministry of Interior, Public Administration and Decentralization; Municipality of Amaroussion; IDHEAP; Austrian Ministry of Public Service and Sports; and Austrian Federal Computer Center.

\section{References}

1. Austrian governmental portal, http://www.help.gv.at

2. Deliverable D231 of eGOV project: GovML syntax and filters implementation

3. Deloitte Research, 2000, 'At the Dawn of e-Government: The Citizen as Customer', available at www.deloitte.com

4. E-envoy home page, http://www.e-envoy.gov.uk/

5. EzGov FlexFoundation, 'Realizing E-Government', White paper, available at http://www.ezgov.com

6. Greek governmental web site, http://www.polites.gr/

7. Hong Kong Special Administrative Region Portal, http://www.esd.gov.hk/

8. ISO/IEC 11179-3, Specification and standardization of data elements, Part 3: Basic attributes of data elements, 1994

9. IST eGov project http://www.egovproject.org

10. Mark Birbeck et al. Professional XML, Wrox Press Ltd, 2001

11. Maria Wimmer, Johanna Krenner. 2001. An Integrated Online One-Stop Government Platform: The eGOV Project. 9th Interdisciplinary Information Management Talks, Proceedings, pp. 329-337 (ISBN 3-85487-272-0)

12. OSCI, the German "de facto" standard for eGovernment, http://www.osci.de

13. Ramon C. Barquin et. Al 2001 "Knowledge Management, the catalyst for e-government", Management concepts.

14. Singapore Government Services, http://www.ecitizen.gov.sg/

15. Swiss governmental portal, http://www.admin.ch/

16. Tambouris, E., Gorilas S., Boukis G., 2001. Investigation of Electronic Government, in: Panhellenic Informatics Conference Workshop Track on "EGoverment", 8-10 November 2001, Zypern

17. U.K governmental portals, http://www.ukonline.gov.uk/, http://www.gateway.gov.uk/

18. United states governmental portal, http://www.firstgov.gov/

19. XSL Transformations (XSLT), http://www.w3.org/TR/xslt 